\title{
A Novel Acellular Dermal Scaffold Prepared Using High-Intensity Focused Ultrasound Energy for the Repair of Soft Tissue Defects
}

\author{
Mohamed M. Abdul-Monem ${ }^{1}$ Mohamed H. Helal ${ }^{2} \quad$ Moustafa N. Aboushelib ${ }^{1}$ \\ ${ }^{1}$ Dental Biomaterials Department, Faculty of Dentistry, Alexandria \\ University, Alexandria, Egypt \\ 2 Periodontology Department, Faculty of Dentistry, Tanta University, \\ Tanta, Egypt \\ Address for correspondence Mohamed M. Abdul-Monem, BDS, \\ MS, PhD, Dental Biomaterials Department, Faculty of Dentistry, \\ Alexandria University, Alexandria 21526, Egypt \\ (e-mail: mohamed.mahmoud@dent.alex.edu.eg).
}

Eur Dent Res Biomater ] 2020;1:45-50

\begin{abstract}
Objective To evaluate a naturally derived acellular dermal scaffold for soft tissue reconstruction using high-intensity focused ultrasound energy (HIFU).

Materials and Methods Acellular dermal scaffolds (ADSs) were prepared by purification of bovine skin. Half of the scaffolds were subjected to high-intensity focused ultrasound energy (HIFU) to modify collagen structure, whereas the other half was used as control. A large skin defect was made in the dorsum of white mice, and the scaffolds were used to cover the induced defects. Wound healing was evaluated histologically after 2, 6, and 12 weeks using common and specific stained sections $(n=20)$.

Statistical Analysis Mean values and standard deviations were calculated for each group, and Student's $t$-test was used for statistical analysis $(\alpha=0.05 ; n=20)$.

Results After 2 weeks, all examined specimens revealed the presence of inflammatory cellular infiltration and early immature blood vessel formation. After 6 weeks, inflammatory cellular infiltration was reduced, with evidence of maturation of new

Keywords

- HIFU

- acellular dermal scaffold

- collagen matrix

- soft tissue repair blood vessels observed for all groups. After 12 weeks, there was a significant increase $(F=124, p<0.01)$ in new collagen formation and count of mature blood vessels observed for the HIFU group compared with control. Evidence of remodeling of new collagen fibers and biodegradation of the grafts was also observed.

Conclusions HIFU-modified ADSs enhanced wound healing and could be used to cover large soft tissue defects.
\end{abstract}

\section{Introduction}

Soft tissue repair and reconstruction is a point of clinical concern in various medical applications. ${ }^{1,2}$ Primary periodontal plastic surgical modalities for soft tissue reconstruction include several types of free connective tissue grafts, which are considered the golden standard. ${ }^{3}$ However, these

DOI https://doi.org/ $10.1055 / \mathrm{s}-0040-1718783$ methods result in donor-site morbidity and increase the risk of postoperative complications such as bleeding, infection, or necrosis. In addition, there are limitations related to the quality and quantity of the harvested tissue. ${ }^{4}$ Soft tissue grafting is perhaps more emphasized during ridge augmentation to ensure proper wound closure and to provide protection of the grafted material. Barrier membranes are also used to prevent and control soft tissue growth during the period
(C) 2020. European Dental Research and Biomaterials Journal

This is an open access article published by Thieme under the terms of the Creative Commons Attribution-NonDerivative-NonCommercial-License, permitting copying and reproduction so long as the original work is given appropriate credit. Contents may not be used for commercial purposes, or adapted, remixed, transformed or built upon. (https://creativecommons.org/licenses/by-nc-nd/4.0/)

Thieme Medical and Scientific Publishers Pvt. Ltd., A-12, 2nd Floor,

Sector 2, Noida-201301 UP, India 
required for ossification of the graft. ${ }^{5}$ Different types of collagen membranes are currently available for guided tissue regeneration. ${ }^{6}$ However, limitations are related to available sizes, shapes, fixation technique, and additional cost. ${ }^{7}$

Type I and type III collagens are the main components of collagen dermal matrices specifically designed for soft tissue regenerative procedures. It consists of a collagen bilayered structure $^{8}$ : the outer is compact macrostructured, which provides occlusive cellular properties and stability-enhancing tissue adherence and favors open healing, whereas the inner is microstructured and consists of a thick, porous, spongy collagen structure that allows blood clot stabilization and provides neoangiogenesis and the ingrowth of soft tissue cells. ${ }^{9}$ In addition to its biocompatibility and lack of immunogenicity, collagen has chemotactic properties that attract fibroblasts, periodontal ligament and gingival fibroblasts, and many other cells, thus allowing better tissue integration.

Acellular dermal scaffolds (ADSs) are obtained from bovine skin and produced by a carefully controlled process that removes the epidermis and dermis cellular content without altering the extracellular matrix (ECM) structure, which provides the basis for cellular ingrowth and subsequent tissue remodeling. ${ }^{10}$ ADS is easily processed to remove antigenic cellular components without causing any damage to the tissue matrix and preserving the structural integrity. The graft could be also loaded with various bioactive mediators to enhance wound healing and tissue remodeling. ${ }^{11}$

High-intensity focused ultrasound energy (HIFU) is currently used to increase collagen remodeling to improve skin texture and vitality. ${ }^{12}$ This energy restructures collagen fibers and increases interbundle spaces, enhancing neocollagenesis and neoelastogenesis in the deep layers of the skin. Additionally, HIFU is currently being tested to enhance the efficacy of drug delivery using nanoparticles as carriers. ${ }^{13}$ The aim of this study was to clinically and histologically evaluate a new ADS prepared using HIFU for the repair of large soft tissue defects in a mouse model.

\section{Materials and Methods}

Healthy adult male albino rats of the Wistar strain weighing 250 to $300 \mathrm{~g}$ (mean weight: $287 \pm 12.34 \mathrm{~g}$ ) and 3 months of age were selected. Animal selection, management, and surgery protocol were approved by the Ethics Research Committee of the Faculty of Dentistry, Alexandria University (IRB no.: 00010556-IORG 0008839). Animals were housed individually in cages and provided with a standard diet for rodents and tap water. Room temperature and humidity were maintained at $23^{\circ} \mathrm{C}$ and $60 \%$, respectively. Animals were acclimatized to approaching and handling for a period of 10 to 15 days before the start of the study $(n=20)$.

\section{Acellular Dermal Scaffold Preparation}

Full-thickness bovine skin specimens $(4 \times 4 \mathrm{~cm})$ were collected and immersed in 5\% acetic acid enriched with $5 \%$ sodium carbonate for 72 hours at $40^{\circ} \mathrm{C}$. Dermal and fatty layers were removed keeping a $0.5-\mathrm{mm}$-thick layer of the dermis. The specimens were then subjected to alkaline-salt treatment by immersion in $5 \%$ sodium hydroxide containing 5\% sodium chloride for 24 hours followed by two freezing and thawing cycles. ${ }^{14}$ The process was repeated two times, and the specimens were washed and neutralized in acetic acid. Specimens were defatted by immersion in a mixture of acetone-chloroform solution for 24 hours.

Half of the specimens received HIFU energy (Ulthera system; Ulthera Inc., Mesa, Arizona, United States) using a 4-MHz probe. A handheld piece delivered an energy level at $7.5 \mathrm{MHz}$ and a focal depth range of 3 to $4.5 \mathrm{~mm}$. A thick layer of ultrasound conducting medium was applied to the specimens, and each nozzle delivered a preset of pulses in a linear pattern at $1-\mathrm{cm}$ intervals delivering an average of 300 shots per specimen. The other half of specimens was used as control. All specimens were washed, air-dried $\left(60^{\circ} \mathrm{C}\right.$ for 100 hours), and finally sterilized by gamma radiation. Specimens were examined using scanning electron microscopy (XL20; Philips, Eindhoven, the Netherlands) to study the internal structure.

\section{Grafting Procedure}

The animals were anesthetized in a chamber supplied with $4 \%$ isoflurane in $100 \%$ oxygen and then maintained through a facemask supplying 1.5 to $2 \%$ isoflurane in $100 \%$ oxygen. The skin over the dorsum was shaved and carefully washed with a mixture of iodine and $70 \%$ ethanol and then one square incision, $3 \times 3 \mathrm{~cm}$, was made on the dorsal-lateral
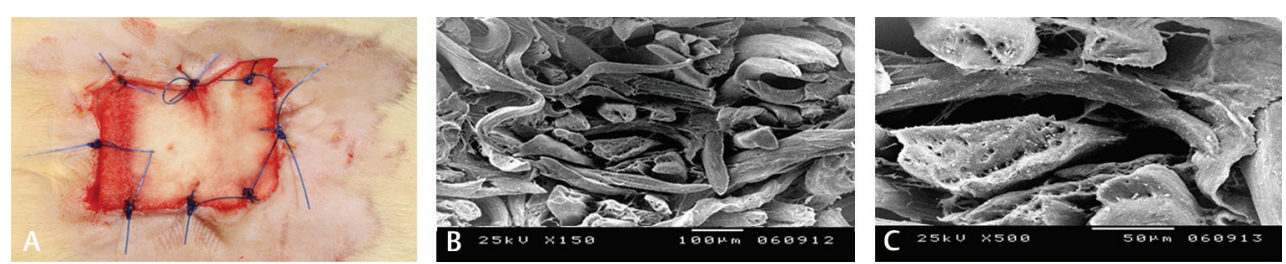

Fig. 1 (A) Digital image showing acellular dermal scaffold (ADS) secured in place on the dorsum of test animal. (B) Scanning electron microscopic image, 150×, showing structure of ADS and separation between collagen bundles. (C) Scanning electron microscopic image, 500×, demonstrating structural relaxation of collagen bundles. 
surface of the mice, approximately $10 \mathrm{~mm}$ above the hip socket. ADS specimens were cut to the required dimension and were rehydrated in sterile saline for 10 minutes. Subsequently, the prepared defect was covered with ADS, which was secured using simple interrupted suture (4-0 Seralon; Resorba, Nuremberg, Germany) ( - Fig. 1A). All the wounds were bandaged properly with paraffin gauze. Broad-spectrum antibiotic (Cefadroxil) and an analgesic (Meloxicam) were administered for 3 consecutive days after the surgery. Animals were sacrificed after 2 weeks, 6 weeks, and 12 weeks in a $\mathrm{CO}_{2}$ euthanasia chamber, according to the ethical guidelines.

\section{Immunohistochemical Analysis}

After fixation in $4 \%$ formaldehyde, each specimen was dehydrated in ascending ethanol concentrations, cleared in xylene, and finally embedded in paraffin. Five- $\mu$ m-thick sections were cut, and representative sections were stained with hematoxylin and eosin and Masson's trichrome stains. Histological assessment was performed under light microscope (Leica ICC50 HD). Immunohistochemical staining for CD34 expression (a marker of angiogenesis) and vimentin expression (a marker of mesenchymal and endothelial cells) was performed. Prepared sections were deparaffinized in xylene and dehydrated in graded alcohol series. To block the internal peroxidase activity, sections were placed in 3\% hydrogen peroxide in a phosphate buffer. Antigen retrieval was achieved in a microwave oven (Panasonic 1380W) for 10 minutes under a pressure of 2 bar. Further incubations with prediluted ready-to-use primary mouse monoclonal antibody, anti-CD34 (QBend 10; DAKO A/S, Glostrup, Denmark), and anti-vimentin specific stains were used as the primary antibody for 30 minutes. Sections were incubated in a moist chamber at room temperature (24 hours) with a working dilution of 1:50 and 1:100 followed by the application of secondary antibody (for 15 minutes), 3,3'-diaminobenzidine (DAB) (to produce brown staining), and Meyer's hematoxylin (for background staining). The specimens were placed in a phosphate buffer saline (PBS) after each mentioned step. The placenta and smooth muscle were used as positive controls for CD34 and vimentin, respectively, according to the manufacturer's instructions. The negative control was obtained by the replacement of primary antibody with PBS. Sections were gradually dehydrated and mounted with coverslips.

The presence of brown-colored reaction localized to the nucleus or the cytoplasm was considered as a positive reaction. The intensity of the immunostaining was classified as negative, weak, or strong from three fields in the blind analysis performed by two pathologists using a conventional light microscope, and further image analysis was performed with an image analysis software (Image J software; version 4.10.03) and a digital camera (Nikon; Tokyo, Japan).

\section{Histomorphometry}

An experienced research associate blindly performed the histomorphometrical analysis and microscopic examination. For image acquisition, a colored digital camera (ColorView III, Olympus, Hamburg, Germany) was mounted on a binocular light microscope (Olympus BX50). Digital images were evaluated using an imaging program (Cell D; Soft Imaging System, Muenster, Germany). Before the histomorphometrical analysis, a calibration procedure was performed to ensure recording repeatable measurements. The following parameters were evaluated descriptively: vascularization of the graft (number of vessels/field), foreign body reaction (thickness of cellular infiltration/field), and amount of new collagen formation (area of stained collagen/filed)

\section{Statistical Analysis}

A statistical analysis software (SPSS version 22.0; IBM Corp., Armonk, New York, United States) was used for data analysis. Mean values and standard deviations were calculated for each group, and Student's $t$-test was used for statistical analysis $(\alpha=0.05 ; n=20)$. Power analysis study indicated that sample size $(n=20)$, a small effect size, and using the selected level of significance resulted in adequate power $(1-\beta=95)$ to detect differences between tested groups.

\section{Results}

All mice were healthy during and after the study, and no complications were observed. All tested collagen scaffolds integrated well into the surrounding soft tissues. Scanning electron microscopic images revealed that HIFU resulted in relaxation of collagen bundles, with microseparation observed in smaller bundles ( - Fig. 1B, 1C).

After 2 weeks of healing, inflammatory cellular infiltration was observed below all specimens. The thickness of cellular infiltration was $45 \mu \mathrm{m}$ for the control and $41 \mu \mathrm{m}$ for HIFU group. Inflammatory cells such as neutrophils, granulocytes, macrophages, and scattered multinucleated giant cells were observed to infiltrate between collagen bundles ADS ( - Fig. 2A). Small immature blood vessel ingrowth was seen as early as 2 weeks, starting at the inner surface of the ADS ( - Fig. 2B). Average number of new blood vessels/field was 1.2 and 1.3 for the control and HIFU group, respectively $(t=6.3 ; p<0.2)$.

After 6 weeks, inflammatory cellular infiltration subsided significantly, $11.2 \mu \mathrm{m}$ for the control and $10.1 \mu \mathrm{m}$ for HIFU group, with evidence of increase in the number of mature blood vessels. Average number of new blood vessels/field was 3.4 and 3.6 for the control and HIFU group, respectively $(t=5.4 ; p<0.32)$ ( - Fig. 3). No evidence of new collagen formation was observed at this period.
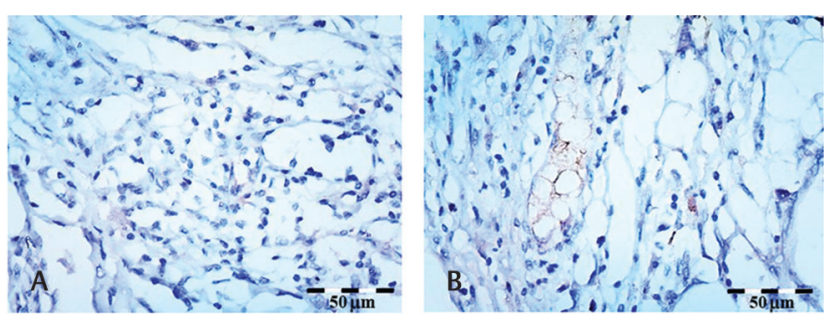

Fig. 2 Stained sections demonstrating (A) inflammatory cellular infiltration and (B) early immature blood vessel formationin acellular dermal scaffold after 2 weeks of surgery. 
After 12 weeks, the HIFU group demonstrated significant increase $(t=14.3 ; p<0.002)$, in mature blood vessel formation (5.3/field) compared with the control (4.2/field). The number of vessels increased and reached the center of HIFU scaffolds, whereas the number of vessels remained stable at the borders. Newly formed connective tissue was observed with evidence of fibroblasts growth and proliferation within collagen bundles. Fibroblasts grew from a monolayer to multiple layers and extended between collagen bundles without any noticeable cellular or morphological changes. New collagen was observed to replace older type after 12 weeks (-Fig. 4). The density of new collagen was significantly higher $(t=19.2 ; p<0.001)$ for the HIFU group (27/field) compared with the control (14.3/field).

Immunohistochemical staining with CD34-positive revealed the presence of microvessel-like structures in the inner layer of collagen matrices after 2 weeks; these microvessels grew toward the central zone after 6 weeks. The mature CD34-positive microvessels with thickened luminal walls were present after 12 weeks. The number of CD34-positive microvessels increased significantly after 12 weeks. Vimentin was strongly and positively expressed in all ADS (-Fig. 5). The difference between tested groups was statistically significant $(F=13.4 ; p<0.001)$ after 12 weeks.
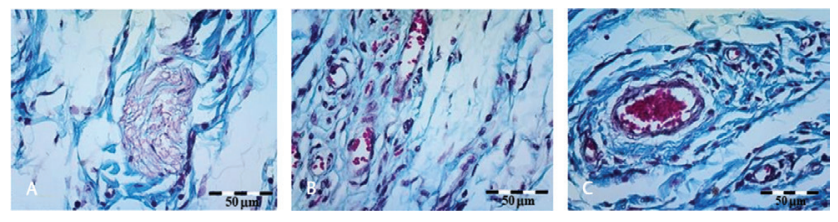

Fig. 3 Stained sections 6 weeks after surgery demonstrating signs of maturation of new blood vessels in the (A) control and (B, C) high-intensity focused ultrasound energy group.
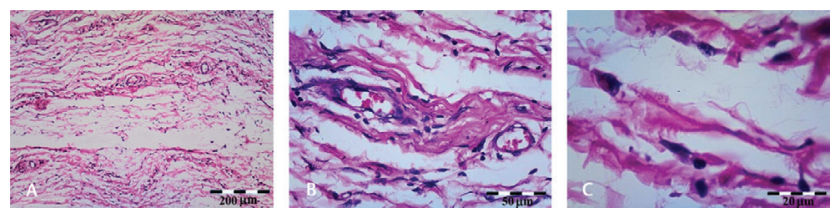

Fig. 4 Stained sections demonstrating collagen reorganization after 12 weeks of healing. (A) Collagen bundles were parallel to the bundles of subcutaneous tissue. (B) Mature formation of blood vessels. (C) Fibroblasts were located between collagen fibers.
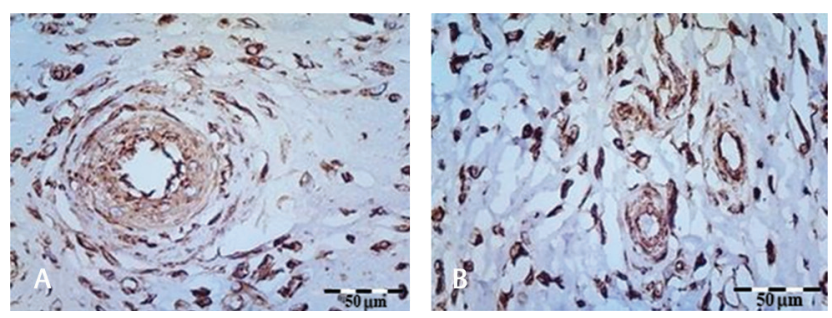

Fig. 5 (A) Immunohistochemical staining with vimentin biomarker showing highly expressed level after 2 weeks, which indicated the presence of myofibroblast-like interstitial cell infiltration. (B) Vimentin positive cells showed progressive increase after 6 weeks.

\section{Discussion}

This study evaluated wound healing of a large skin defect using acellular collagen scaffold prepared using HIFU applied to produce a modified collagen structure. Scanning electron microscopic images revealed that the dense collagen bundles were separated by interbundle spaces and within-bundle spaces. This modified structure enhanced handling and shaping of the graft and allowed accurate fixation without fear of tearing or rupture. Additionally, loosening of collagen bundles allowed enhanced fluid circulation, cell migration, and restructuring of the scaffold in relation to the wound area.

Angiogenesis is a basic requirement for wound healing and establishes an ideal environment for migration and multiplying of endothelial cells from preexisting vessels..$^{15}$ Examination of early wound healing revealed characteristic initial inflammatory cellular reaction followed by early vascularization and new blood vessel formation, which encouraged early remodeling of the scaffolds. Compared with the controls, a higher number of newly formed blood vessels reached significant level as early as 12 weeks. Moreover, new blood vessels were able to reach the center of the scaffolds, which is a positive sign for early tissue integration. Immunohistochemical staining revealed early stages of neoangiogenesis and further steps of vessel maturation. More blood vessels were observed in the inner sections compared with the central sections. This observation is in accordance with a previous experimental study performed by Thoma et al, who reported similar findings. ${ }^{16}$ This findings can be explained by previous experimental study, which indicated that exposure to HIFU can increase the expression of collagen and integrins of ECM-related molecules that promote the formation of a large number of vessellike structure and capillaries. ${ }^{17}$

Early invasion of fibroblasts is one of the advantages of collagen scaffolds. ${ }^{18}$ New collagen formation and tissue organization is a sensitive step requiring balance between the rate of biodegradation and the rate of new tissue formation to prevent tissue collapse during early phases of healing. Similarly, prevention of excessive fibrous tissue deposition should be controlled to prevent scar formation and wound contraction. Immunohistochemical staining with vimentin biomarkers showed highly expressed levels after 2 weeks of surgery, which confirms the presence of myofibroblast-like interstitial cell infiltration. Vimentin positive cells showed progressive increase after 12 weeks for the HIFU group, indicating the role of HIFU in enhancing the structure of scaffolds.

Moreover, the present histological findings showed that ADS did not initiate excessive immunological or foreign body reactions during early wound healing. ${ }^{19}$ On the contrary, HIFU is known to reduce these reactions, which improved the quality and normality of inflammatory reactions. ${ }^{20-22}$ Although few inflammatory cells and multinuclear giant cells were detected, a previous study demonstrated that native collagen matrix and dehydrothermally cross-linked matrix were associated with multinucleated cellular infiltration observed between the collagen bundles. 
The study reported that a native porcine collagen matrix seems to be the best option for soft tissue augmentation procedures even if the volume stability is lower than with non-crosslinked collagen. ${ }^{23}$

In contrast to previous studies, ${ }^{24,25}$ it was observed that multinucleated giant cells between the collagen fibers acted as a source of vascular-inducing signaling molecules, such as vascular endothelial growth factor, and contributed to proliferation of granulation tissue within the structure of the scaffolds. Cultivating scaffolds with endothelial and mesenchymal cells might improve cell-to-cell communication with the surrounding host tissue, which would eventually improve scaffold integration and biodegradation. ${ }^{24}$ Barbeck et a ${ }^{26}$ have shown that the blood vessel framework and the presence of fatty tissue islands allowed connective tissue proliferation into the ADS scaffolds and enabled microvessels to infiltrate the collagenous structure. This specific vascularization pattern is different from vascularization accompanying multinucleated cell triggered inflammatory process, in which vessels spread out as a response to biodegradation of cellular content. ADSs were easily adapted and secured to cover the entire defect area, and new hair growth was observed to almost completely cover the wound area after 12 weeks. Longer observation periods are required to study the long-term behavior of ADS.

\section{Conclusions}

Within the limitations of this study, HIFU improved integration and biodegradation of ADSs. After 12 weeks, the amount of new collagen and mature blood vessels formed in the surgical defect covered by the HIFU ADS significantly increased compared with the control group. HIFU modified ADS enhanced wound healing and could be used to cover large soft tissue defects.

\section{Ethical Approval}

This study was approved by the Ethics Research Committee of the Faculty of Dentistry, Alexandria University (IRB no.: 00010556-IORG 0008839).

\section{Authors' Contributions}

All authors contributed equally to this research and the manuscript.

\section{Conflict of Interest}

None declared.

\section{References}

1 Coraux C, Hilmi C, Rouleau M, et al. Reconstituted skin from murine embryonic stem cells. Curr Biol 2003;13(10):849-853

2 De Filippo RE, Yoo JJ, Atala A. Urethral replacement using cell seeded tubularized collagen matrices. J Urol 2002;168(4 Pt 2): 1789-1792, discussion 1792-1793

3 Moraschini V, de Almeida DCF, Sartoretto S, Bailly Guimarães H, Chaves Cavalcante I, Diuana Calasans-Maia M. Clinical efficacy of xenogeneic collagen matrix in the treatment of gingival recession: a systematic review and meta-analysis. Acta Odontol Scand 2019;77(6):457-467
4 Murphy KG, Gunsolley JC. Guided tissue regeneration for the treatment of periodontal intrabony and furcation defects. A systematic review. Ann Periodontol 2003;8(1):266-302

5 Zafar M, Najeeb S, Khurshid Z, et al. Potential of electrospun nanofibers for biomedical and dental applications. Materials (Basel) 2016;9(2):73

6 Sheikh Z, Najeeb S, Khurshid Z, Verma V, Rashid H, Glogauer M. Biodegradable materials for bone repair and tissue engineering applications. Materials (Basel) 2015;8(9):5744-5794

7 Park YH, Choi SH, Cho KS, Lee JS. Dimensional alterations following vertical ridge augmentation using collagen membrane and three types of bone grafting materials: a retrospective observational study. Clin Implant Dent Relat Res 2017;19(4):742-749

8 Boyce ST, Supp AP, Swope VB, Warden GD. Vitamin C regulates keratinocyte viability, epidermal barrier, and basement membrane in vitro, and reduces wound contraction after grafting of cultured skin substitutes. J Invest Dermatol 2002;118(4):565-572

9 Tucker WD, Arora Y, Mahajan K, Anatomy, Blood Vessels. Treasure Island, FL: StatPearls Publishing; 2020

10 Halper J, Kjaer M. Basic components of connective tissues and extracellular matrix: elastin, fibrillin, fibulins, fibrinogen, fibronectin, laminin, tenascins and thrombospondins. Adv Exp Med Biol 2014;802:31-47

11 Bondioli E, Fini M, Veronesi F, et al. Development and evaluation of a decellularized membrane from human dermis. J Tissue Eng Regen Med 2014;8(4):325-336

12 Suh DH, ChoiJH, Lee SJ, Jeong KH, Song KY, Shin MK. Comparative histometric analysis of the effects of high-intensity focused ultrasound and radiofrequency on skin. J Cosmet Laser Ther 2015;17(5):230-236

13 Lee $\mathrm{S}$, Han $\mathrm{H}$, Koo H, et al. Extracellular matrix remodeling in vivo for enhancing tumor-targeting efficiency of nanoparticle drug carriers using the pulsed high intensity focused ultrasound. J Control Release 2017;263:68-78

14 Gorlov IF, Titov EI, Semenov GV, et al. Collagen from porcine skin: a method of extraction and structural properties. Int J Food Prop 2018;21(1):1031-1042

15 Tonnesen MG, Feng X, Clark RA. Angiogenesis in wound healing. J Investig Dermatol Symp Proc 2000;5(1):40-46

16 Thoma DS, Villar CC, Cochran DL, Hämmerle CH, Jung RE. Tissue integration of collagen-based matrices: an experimental study in mice. Clin Oral Implants Res 2012;23(12):1333-1339

$17 \mathrm{Mu}$ HM, Wang LY. Effect of therapeutic ultrasound on brain angiogenesis following intracerebral hemorrhage in rats. Microvasc Res 2015;102:11-18

18 Lima RS, Peruzzo DC, Napimoga MH, Saba-Chujfi E, Dos Santos-Pereira SA, Martinez EF. Evaluation of the biological behavior of Mucograft $₫$ in human gingival fibroblasts: an in vitro study. Braz Dent J 2015;26(6):602-606

19 Watson T. Ultrasound in contemporary physiotherapy practice. Ultrasonics 2008;48(4):321-329

20 Carrer VM, Setti JAP, Veronez DL, Moser AD. Continuous therapeutic ultrasound in the healing process in rat skin. Fisioter Mov 2015;28(4):751-758

21 Mortimer AJ, Dyson M. The effect of therapeutic ultrasound on calcium uptake in fibroblasts. Ultrasound Med Biol 1988;14(6):499-506

22 Nussbaum EL, Locke M. Heat shock protein expression in rat skeletal muscle after repeated applications of pulsed and continuous ultrasound. Arch Phys Med Rehabil 2007;88(6): 785-790

23 Rothamel D, Benner M, Fienitz T, et al. Biodegradation pattern and tissue integration of native and cross-linked porcine collagen soft tissue augmentation matrices - an experimental study in the rat. Head Face Med 2014;10:10 
24 Silva SS, Popa EG, Gomes ME, et al. Silk hydrogels from non-mulberry and mulberry silkworm cocoons processed with ionic liquids. Acta Biomater 2013;9(11):8972-8982

25 Leal AI, Caridade SG, Ma J, et al. Asymmetric PDLLA membranes containing Bioglass ${ }^{\circledR}$ for guided tissue regeneration: characterization and in vitro biological behavior. Dent Mater 2013;29(4):427-436
26 Barbeck M, Lorenz J, Kubesch A, et al. Porcine dermis-derived collagen membranes induce implantation bed vascularization via multinucleated giant cells: a physiological reaction? J Oral Implantol 2015;41(6):e238-e251 